\title{
Slow Progress and Persistent Challenges for the Underrepresented Minority Family Physician
}

\author{
Kendall M. Campbell, MD
}

(J Am Board Fam Med 2018;31:840-841.)

In the article, "Board certified family physician workforce: progress in racial and ethnic diversity," the authors review the racial and ethnic background of physicians to document proportional growth over time as compared with 2017 United States Census data. In their analysis, they aggregated data provided by family medicine physicians at the time of application for their American Board of Family Medicine (ABFM) certification examination. The years evaluated were from 2013 to 2017 , and that timespan yielded a match of 66,542 board certified family physicians for the study. Results showed that the growth of the number of board certified family medicine black and Hispanic or Latino physicians lags behind other groups, as well as census defined numbers, $7.8 \%$ versus $13.3 \%$ for black physicians and $9.1 \%$ versus $17.8 \%$ for Hispanic or Latino physicians. ${ }^{1}$ Parity was seen with white physicians (61.2\% vs $61.3 \%$ ), and Asian physicians $(21.8 \%$ vs $5.7 \%)$ were overrepresented as compared with their representation in the US population. Even though there has been an overall increase in physician numbers over the past 30 years, the numbers of black and Hispanic or Latino physicians has not kept the pace.

These data are consistent with other studies that cross specialty and geography showing the need for growth of underrepresented in medicine minorities (URMM). ${ }^{2,3}$ Data further highlight the need for increasing numbers to better reflect the composition of the general population. The reasons for inertia in URMM representation are multifactorial and include the persistence of systems designed to

From Brody School of Medicine, East Carolina University, Greenville, NC.

Funding: none.

Conflict of interest: none declared.

Corresponding author: Kendall M. Campbell, MD, Office of Diversity Affairs, 600 Moye Blvd 1N-16, Greenville, NC 27834 (E-mail: campbellke16@ecu.edu). separate and divide based on class, social status, or race. ${ }^{4}$ In addition, bias against minorities, mistreatment of minority groups, and the promotion of the white race over others has escalated under the current presidential leadership, and these issues have added to the problem. ${ }^{5,6}$ Another reason includes the out of proportion increases to the pool of URMM physicians from historically minority-serving medical schools as compared with their predominantly white institution counterparts, ${ }^{7,8}$ which are more numerous in number and often better resourced. The "minority tax" that impacts the URMM faculty member directly impacts the recruitment and retention of these groups in academic medicine. The minority tax has also been a tough challenge to overcome and includes lack of mentorship, lack of faculty development, isolation, diversity pressures, and racism, ${ }^{9}$ impacting recruitment and retention of URMM faculty in academic medicine.

The gravity of decreased numbers of URMM physicians has to be articulated. Family medicine is a primary care leader in racial/ethnic physician diversity, ${ }^{10}$ and limited diversity in the specialty impacts care for underserved populations, threatening the health of families and communities. Added to this concern would be a direct hindrance toward achieving the triple aim of reducing per capita health care costs, improving the care experience, and improving the health of populations. Physicians who mirror the composition of the community have the potential to improve all three aims, potential that cannot be ignored as the health care system moves from a focus on disease to a focus on health.

What is needed to promote equity for URMM and increase their numbers in medicine? How do we increase numbers of board certified black and Hispanic or Latino physicians? Where to start has been a challenge, and increasing awareness is a must. The National Academy of Medicine as well 
as the Associate of American Medical Colleges have championed the push to increase numbers of URMMs. Approaches that have been helpful to increase URMM presence, starting often at the precollege level, include pipeline and outreach programs. These programs are designed to increase exposure to health care and enhance academic ability and can be effective at increasing numbers of URMM. ${ }^{11,12}$ Continued exploration of community colleges as a pathway for URMM should help increase numbers, as there are data that suggest the community college path may be a route to family medicine. ${ }^{13,14}$ Additional resources and support should be provided to undergraduate and medical schools that demonstrate evidence of increasing URMMs. Consideration should be given to creating new medical schools and residency training programs at historically black colleges and universities. Even though there is some evidence that family medicine residency programs are becoming more diverse, ${ }^{15}$ additional focus on making the residency training environment more diverse and culturally welcoming is needed.

In order for true equity to be seen in family medicine for minority family medicine physicians, systems of inequity, bias, and discrimination that propagate separation and division have to be dismantled. There is much ground to cover to increase numbers of underrepresented groups in medicine and to continue promoting equity for these groups. The future of health care for families and communities depend on it.

To see this article online, please go to: bttp://jabfm.org/content/ 31/6/840.full.

\section{References}

1. Peabody MR, Eden AR, Douglas M, Phillips RL. Board certified family physician workforce: progree in racial and ethnic diversity. J Am Board Fam Med 2018;31:842-56.

2. Yu PT, Parsa PV, Hassanein O, Rogers SO, Chang DC. Minorities struggle to advance in academic medicine: a 12-y review of diversity at the highest levels of America's teaching institutions. J Surg Res 2013;182:212-8.
3. Walker KO, Moreno G, Grumbach K. The association among specialty, race, ethnicity, and practice location among California physicians in diverse specialties. J Natl Med Assoc 2012;104:46-52.

4. Jones CP. Systems of power, axes of inequity: parallels, intersections, braiding the strands. Med Care 2014;52:S71-5.

5. Bobo LD. Racism in Trump's America: reflections on culture, sociology, and the 2016 US presidential election. Br J Sociol 2017;68:S85-S104.

6. Lamont M, Park BY, Ayala-Hurtado E. Trump's electoral speeches and his appeal to the American white working class. Br J Sociol 2017;68:S153-S80.

7. Rodriguez JE, Lopez IA, Campbell KM, Dutton M. The role of historically black college and university medical schools in academic medicine. J Health Care Poor Underserved 2017;28:266-78.

8. Mader EM, Rodriguez JE, Campbell KM, et al. Status of underrepresented minority and female faculty at medical schools located within Historically Black Colleges and in Puerto Rico. Med Educ Online 2016;21:29535.

9. Rodriguez JE, Campbell KM, Pololi LH. Addressing disparities in academic medicine: what of the minority tax? BMC Med Educ 2015;15:6.

10. Campbell KM, Rodriguez JE. Could more black doctors being in primary care specialties be the result of institutional racism? Acad Med 2018;93:346.

11. Campbell KM, Berne-Anderson T, Wang A, Dormeus G, Rodriguez JE. USSTRIDE program is associated with competitive Black and Latino student applicants to medical school. Med Educ Online 2014;19:24200.

12. Campbell KM, Brownstein NC, Livingston H, Rodriguez JE. Improving underrepresented minority in medicine representation in medical school. South Med J 2018;111:203-8.

13. Talamantes E, Mangione CM, Gonzalez K, Jimenez A, Gonzalez F, Moreno G. Community college pathways: improving the U.S. physician workforce pipeline 2014;89:1649-56.

14. Talamantes E, Jerant A, Henderson MC, et al. Community college pathways to medical school and family medicine residency training. Ann Fam Med 2018; 16:302-7.

15. Xierali IM, Hughes LS, Nivet MA, Bazemore AW. Family medicine residents: increasingly diverse, but lagging behind underrepresented minority population trends. Am Fam Physician 2014;90:80-1. 